\title{
RGD-Dendrimer-Poly(L-lactic) Acid Nanopatterned Substrates for the Early Chondrogenesis of Human Mesenchymal Stromal Cells Derived from Osteoarthritic and Healthy Donors
}

\author{
Cristina Rodríguez-Pereira ${ }^{1,2}{ }^{\oplus}$, Anna Lagunas ${ }^{3,4}{ }^{\oplus}$, Ignasi Casanellas ${ }^{3,4,5}{ }^{(D}$, Yolanda Vida ${ }^{6,7}(\mathbb{D}$, \\ Ezequiel Pérez-Inestrosa 6,7 (D), José A. Andrades ${ }^{3,8}$, José Becerra ${ }^{3,7,8}$, Josep Samitier ${ }^{3,4,5}$, \\ Francisco J. Blanco 1,2,9 (D) and Joana Magalhães ${ }^{1,2,3, *(D)}$ \\ 1 Unidad de Medicina Regenerativa, Grupo de Investigación en Reumatología (GIR), Instituto de Investigación \\ Biomédica de A Coruña (INIBIC), Complejo Hospitalario Universitario de A Coruña (CHUAC), Sergas, \\ 15006 A Coruña, Spain; cristina.rodriguez.pereira@sergas.es (C.R.-P.); fblagar@sergas.es (F.J.B.) \\ 2 Centro de Investigaciones Científicas Avanzadas (CICA), Universidade da Coruña (UDC), \\ As Carballeiras S/N, Campus de Elviña, 15071 A Coruña, Spain \\ 3 Networking Biomedical Research Center in Bioengineering, Biomaterials and Nanomedicine (CIBER-BBN), \\ 28029 Madrid, Spain; alagunas@ibecbarcelona.eu (A.L.); icasanellas@ibecbarcelona.eu (I.C.); \\ andrades@uma.es (J.A.A.); becerra@uma.es (J.B.); jsamitier@ibecbarcelona.eu (J.S.) \\ 4 Institute for Bioengineering of Catalonia (IBEC), Barcelona Institute of Science and Technology (BIST), \\ 08028 Barcelona, Spain \\ 5 Department of Electronics and Biomedical Engineering, University of Barcelona (UB), 08028 Barcelona, Spain \\ 6 Dpto. Química Orgánica, Universidad de Málaga-IBIMA, Campus de Teatinos s/n, 29071 Málaga, Spain; \\ yolvida@uma.es (Y.V.); inestrosa@uma.es (E.P.-I.) \\ 7 Centro Andaluz de Nanomedicina y Biotecnología (BIONAND), Parque Tecnológico de Andalucía, \\ C/Severo Ochoa, 35, 29590 Campanillas, 29590 Málaga, Spain \\ 8 Cell Biology, Genetics and Physiology Department, Instituto de Investigación Biomédica de Málaga (IBIMA), \\ University of Malaga (UMA), 29071 Málaga, Spain \\ 9 Departamento de Medicina, Facultad Ciencias de la Salud, Campus de Oza, Universidade da Coruña (UDC), \\ Campus de Oza, 15006 A Coruña, Spain \\ * Correspondence: joana.cristina.silva.magalhaes@sergas.es; Tel.: +34-981-176-413
}

Received: 17 April 2020; Accepted: 12 May 2020; Published: 13 May 2020

\begin{abstract}
Aiming to address a stable chondrogenesis derived from mesenchymal stromal cells (MSCs) to be applied in cartilage repair strategies at the onset of osteoarthritis (OA), we analyzed the effect of arginine-glycine-aspartate (RGD) density on cell condensation that occurs during the initial phase of chondrogenesis. For this, we seeded MSC-derived from OA and healthy (H) donors in RGD-dendrimer-poly(L-lactic) acid (PLLA) nanopatterned substrates (RGD concentrations of $4 \times 10^{-9}, 10^{-8}, 2.5 \times 10^{-8}$, and $\left.10^{-2} \mathrm{w} / \mathrm{w}\right)$, during three days and compared to a cell pellet conventional three-dimensional culture system. Molecular gene expression (collagens type-I and II-COL1A1 and COL2A1, tenascin-TNC, sex determining region Y-box9-SOX9, and gap junction protein alpha 1-GJA1) was determined as well as the cell aggregates and pellet size, collagen type-II and connexin 43 proteins synthesis. This study showed that RGD-tailored first generation dendrimer (RGD-Cys-D1) PLLA nanopatterned substrates supported the formation of pre-chondrogenic condensates from OA- and H-derived human bone marrow-MSCs with enhanced chondrogenesis regarding the cell pellet conventional system (presence of collagen type-II and connexin 43 , both at the gene and protein level). A RGD-density dependent trend was observed for aggregates size, in concordance with previous studies. Moreover, the nanopatterns' had a higher effect on OA-derived MSC morphology, leading to the formation of bigger and more compact aggregates with improved expression of early chondrogenic markers.
\end{abstract}


Keywords: cell condensation; gap junctions; RGD-density; chondrogenic differentiation; osteoarthritis

\section{Introduction}

Osteoarthritis $(\mathrm{OA})$ is a degenerative disease affecting the articular cartilage present at the movable joints, being characterized by cell stress and extracellular matrix (ECM) degradation, initiated by micro- and macro-injuries that activate maladaptive repair responses including pro-inflammatory pathways [1]. Given that nowadays there is still no cure, a need for clinical solutions is imperative. Regenerative medicine therapies for OA treatment, based on mesenchymal stromal cells (MSCs) and biomaterials have been of focus since the late 90s, and even though significant advances have been made, a successful guidance of the MSC chondrogenic differentiation process towards a stable adult chondrocyte phenotype has not yet been fully achieved [2,3].

Controlled nanoscaled topographies or surface nanopatterning have been proposed as potential tools for cell behavior guidance including adhesion, proliferation, and differentiation towards specific lineages via the regulation of membrane proteins $[4,5]$. Specific cell adhesion can be achieved via the formation of focal adhesions and triggered by the bioconjugation of ligands present in the ECM, such as fibronectin, and more specifically the arginine-glycine-aspartate (RGD) sequence, to the corresponding cell receptor, integrin [6]. Most research on the effect of RGD nanopatterns on MSC guidance has been focused on osteogenesis and adipogenesis, demonstrating the role of RGD nanospacing as an inherent regulator of stem cell differentiation [4,6-8]. On the other hand, fewer studies can be found on the effect of nanopattern tuning on MSC chondrogenesis, albeit these indicate that both nanopattern distribution and composition of group functionality can regulate the chondrogenic process $[9,10]$.

Dendrimer nanopatterning is a bottom-up nanopatterning technique based on the self-assembly of absorbed dendrimers in low-charged surfaces in liquid-like order [11]. This technique allows the nanoscale control of surface functionalization on large areas, thus being fully compatible with cell culture procedures. In previous work, we have synthesized RGD-tailored first generation dendrimers (RGD-Cys-D1) that incorporate the fibronectin tripeptide sequence RGD, responsible for integrin-mediated cell adhesion [12]. Since dendrimers are $4-5 \mathrm{~nm}$ in diameter [12], although presenting up to eight copies of RGD, only one integrin (approximately $10 \mathrm{~nm}$ diameter) [13] will interact per dendrimer. Thereby, nanopattern configurations correspond to the available RGD distribution for cell adhesion. Nanopatterns of a range of biologically relevant local surface densities were thus obtained from different RGD-Cys-D1 initial concentrations in aqueous solution deposited on poly(L-lactic) acid (PLLA) substrates [12,14]. In this work, we have shown an association between local RGD surface density and adipose tissue (AT)-derived MSC chondrogenic commitment, demonstrating that an intermediate adhesiveness of cells resulted in larger cell condensates with enhanced early chondrogenic differentiation [14].

Condensation is a prerequisite for chondrogenic differentiation. In this cellular aggregation stage, cellular communication occurs determined by the expression of cell-cell adhesion molecules such as $\mathrm{N}$-cadherin, neural cell adhesion molecule, or gap junctions [15]. Thus, these molecules, together with the ECM, must be strictly controlled to determine the size and shape of the condensations [16]. A high percentage of these cell-to-cell interactions are channels of the gap-junction (GJ) type, specifically those composed by connexin 43 (expressed by the gap junction protein alpha 1, GJA1) [17,18]. This protein has been described as being implicated in a great number of processes including chondrogenesis as well as in the pathogenesis of osteoarthritis [19]. In particular, our group was the first to demonstrate the expression of several GJ proteins in human cartilage as well as altered levels of different connexins including overexpression of GJA1 in OA-derived tissue [20].

Later, Schrobback et al. investigated the influence of gap junction-mediated intercellular contacts on bone marrow derived-MSCs chondrogenesis on alginate and microtissue models (restricted vs. 
induced cell-cell contacts), showing that a reduction in direct cell-cell contacts did not affect the chondrogenic process, however, blocking gap junctions compromised cell differentiation [21].

Hereby, this study was aimed to compare the chondrogenic differentiation potential of human BM-MSCs derived from OA or healthy $(\mathrm{H})$ donors in uneven RGD-Cys-D1-PLLA nanopatterned substrates using a three-dimensional (3D) pellet culture system as a reference model to mimic prechondrogenic condensation, with a focus on the influence of gap-junction mediated cell-to-cell interactions in the chondrogenic differentiation process.

\section{Materials and Methods}

\subsection{RGD-Cys-D1-Dendrimer PLLA Nanopatterned Substrate Production}

A $2 \% \mathrm{~m} / \mathrm{v}$ 95/5 L-lactide/DL-lactide copolymer solution (PLLA) (Corbion, Barcelona, Spain) in dry 1,4-dioxane (Panreac, Madrid, Spain) was deposited on $1.25 \times 1.25 \mathrm{~cm}^{2}$ Corning glass microslides (Sigma-Aldrich, Madrid, Spain) and spin-coated at $3000 \mathrm{rpm}$ for $30 \mathrm{~s}$ (Laurell Tech. Corp., North Wales, UK). Surface nanopatterning was conducted by immersing the spin-coated PLLA substrates in aqueous solutions of RGD-Cys-D1, for $16 \mathrm{~h}(\mathrm{pH}=5.6, \mathrm{~T}=293 \mathrm{~K})$, at the concentration of $4 \times 10^{-9}$, $10^{-8}, 2.5 \times 10^{-8}$, and $10^{-2} \mathrm{w} / \mathrm{w}$, followed by copious rinsing with water, as previously described [11]. Deionized water (18 M $\Omega \cdot \mathrm{cm}^{-1}$ Milli-Q, Millipore, Madrid) was used to prepare all solutions and for sample rinsing. All solutions were sonicated and filtered (Millex RB sterile filter, Merck Millipore, Madrid, Spain) prior to use. Ultraviolet light (UV) exposure for $15 \mathrm{~min}$ was used for sterilization before cellular experiments.

\subsection{Bone Marrow-Mesenchymal Stromal Cells Isolation and Chondrogenic Differentiation}

Human mesenchymal stromal cells (MSCs) were isolated from the bone marrow (BM) of femur heads obtained from seven patients (six females and one male, with a mean age of $88 \pm 4.3$ ), of which, four were osteoarthritic $(\mathrm{OA})$ and three healthy $(\mathrm{H})$, as classified by qualified clinical physicians, according to the Kellgren and Lawrence (K-L) radiological grading system [22]. The patients signed an informed consent agreement form prior to collection. The study was conducted in accordance with the Declaration of Helsinki and the protocol approved by the Galician Research Ethics Committee (Project Registry Code: 2014/019).

BM-MSCs were cultured in a basal medium (BM) composed by Dulbecco's modified Eagle's medium (DMEM, Lonza, Porriño, Spain) supplemented with 20\% fetal bovine serum (Gibco, Madrid, Spain) and penicillin/streptomycin (10,000 IU/mL) (Gibco, Madrid, Spain) until 90\% confluent. Pre-plating technique was performed to avoid remaining fibroblasts [23]. Cells used in all experiments were mycoplasma-free.

For chondrogenic differentiation studies (Figure 1), BM-MSCs derived from OA and $\mathrm{H}$ patients were seeded separately in a 12-well culture plate at densities of $5 \times 10^{3}$ (for immunofluorescence) or $2.5 \times 10^{5}$ (for gene expression analysis) cells per well, in RGD-Cys-D1 PLLA nanopatterned substrates, at different dendrimer concentrations $\left(4 \times 10^{-9}, 10^{-8}, 2.5 \times 10^{-8}\right.$, and $\left.10^{-2} \mathrm{w} / \mathrm{w}\right)$. Fibronectin-coated PLLA (Fn-PLLA) $(100 \mu \mathrm{g} / \mathrm{mL})$ and untreated-PLLA were used as positive and negative controls, respectively.

Cells were incubated in a well-defined chondrogenic differentiation medium (CM) composed of DMEM supplemented with knockout serum (15\%, Invitrogen, Barcelona, Spain), ascorbic acid $(10 \mu \mathrm{L} / \mathrm{mL})$, transferrin $(6 \mu \mathrm{L} / \mathrm{mL})$, dexamethasone $(10 \mu \mathrm{M})$, retinoic acid $\left(10^{-7} \mathrm{M}\right)$ (all from Sigma-Aldrich, Madrid, Spain), and transforming growth factor beta 3 (10 ng/mL, Prospec-Tany TechnoGene, Deltaclon, Madrid, Spain), at $37^{\circ} \mathrm{C}$ under $5 \% \mathrm{CO}_{2}$ for three days. Cells seeded in Fn-PLLA under basal medium conditions were used as a negative control of chondrogenesis.

Finally, conventional 3D pellet culture was used as a reference model for chondrogenesis [24]. Briefly, $2.5 \times 10^{5}$ cells, suspended in $500 \mu \mathrm{L} \mathrm{CM}$, were centrifuged at $600 \mathrm{~g}$, for $10 \mathrm{~min}$, in $15 \mathrm{~mL}$ polypropylene conical tubes. Pellet-cells were incubated in the same conditions as our experimental model. 


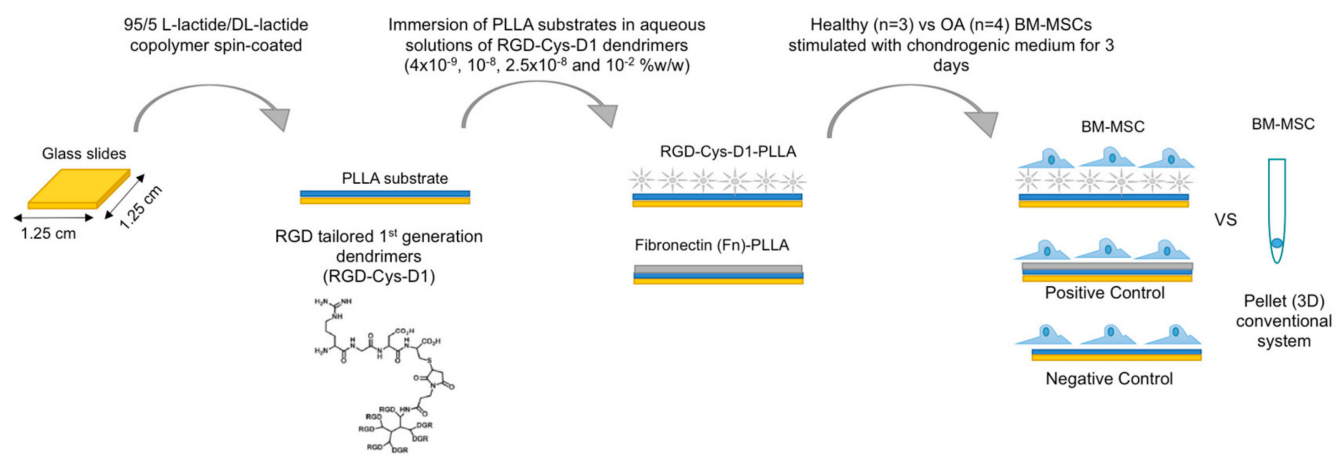

Figure 1. Schematic representation of the RGD-Cys-D1 PLLA nanopatterned substrates preparation process and cell seeding.

\subsection{Immunofluorescence and Histology}

PLLA-cell substrates were fixed in $4 \%$ (w/v) paraformaldehyde (Sigma-Aldrich, Madrid, Spain). BM-MSCs pellets were frozen in optimal cutting temperature (OCT) embedding matrix (Sakura, Barcelona, Spain). Immunofluorescence labeling was performed for collagen type-II (col-II) (1:50; Abcam, UK) and Cx43 (1:50; BD, Madrid, Spain). Fluorescein isothiocyanate (FITC) (1:10; DAKO, Barcelona, Spain) and ribulose-5-phosphate-3-epimerase (RPE) (1:20; DAKO, Barcelona, Spain) were respectively used as secondary antibodies, and 4',6-diamidino-2-phenylindole (DAPI) (Sigma-Aldrich, Madrid, Spain) as counterstaining. Photographs were performed using the Olympus microscope (BX61, Olympus, Japan). Hematoxylin-Eosin (HE) and Safranin-O (SO) staining of the BM-MSCs pellets were performed in order to assess cell morphology and proteoglycan synthesis, respectively.

\subsection{BM-MSC Aggregates and Pellet Areas}

The area of BM-MSC aggregates formed in each experimental condition was calculated using ImageJ (Fiji version, U.S. National Institute of Health, Bethesda, MD, USA) [25] considering a cell aggregate as a group of at least five cells. The analysis was performed manually by selecting the cell aggregates (Selection tool) and determining their area with the Measure tool in fluorescence images of cells stained with DAPI. The results were presented as the mean of at least three cell aggregates. The area of each pellet formed using the conventional method was calculated following the same methodology, but using HE-stained micrographs. The results were presented as a mean of four OA- and three H-MSCs derived pellets, respectively.

\subsection{Molecular Analysis}

Total RNA $(1 \mu \mathrm{g})$ was isolated from both PLLA-cell substrates and cell pellets, previously pulverized in a Mikro-Dismembrator (Retsch MM200, Retsch GmBH, Hann, Germany) using the Trizol method (Invitrogen, Madrid, Spain). RNA samples were treated with DNase and converted into cDNA using the SuperScript VILO cDNA synthesis kit (Invitrogen, Madrid, Spain).

Gene expression was measured by real-time quantitative reverse transcription-polymerase chain reaction (qRT-PCR) conducted in a LightCycler 480 Instrument (Roche, Madrid, Spain) using the LightCycler 480 Probes Master protocol. Amplification of mRNA was performed using custom-made primers for gap junction protein alpha 1 (GJA1), collagen type II (COL2A1), sex determining region Y-box9 (SOX9), tenascin (TNC), and collagen type I (COL1A1). RPL13a was used as the housekeeping gene (HKG) (Table 1).

The amplification program consisted of an initial denaturation at $95^{\circ} \mathrm{C}$ for $10 \mathrm{~min}$, followed by 45 cycles at $95^{\circ} \mathrm{C}$ for $10 \mathrm{~s}$, annealing at $60^{\circ} \mathrm{C}$ for $30 \mathrm{~s}$, and an extension at $72{ }^{\circ} \mathrm{C}$ for $1 \mathrm{~s}$. Quantitative data were performed using qbase + software, version 3.1 (Biogazelle, Zwijnaarde, Belgium). Data were normalized against the expression values obtained for the positive control (Fn-PLLA) in healthy donor cells for each gene, which was assigned the value of 1 , and measured as relative expression levels. 
Table 1. Custom made primers.

\begin{tabular}{ccccl}
\hline Genes & Forward & Reverse & Probe & $\begin{array}{c}\text { GeneBank } \\
\text { Accession } \mathbf{n}^{\circ}\end{array}$ \\
\hline CX43 & gcctgaacttgcctttcat & ctccagtcacccatgttgc & 88 & NM_000165.4 \\
\hline COL2A1 & tggtgctaatggcgagaag & cccagtctctccacgttcac & 4 & NM_001844.4 \\
\hline SOX9 & gtaccegcacttgcacaac & tcgctctcgttcagaagtctc & 61 & NM_000346.3 \\
\hline COL1A1 & ctggcccattggtaatgt & accagggaaaccagtagcac & 1 & NM_000088.3 \\
\hline TNC & ggtacagtgggacagcaggt & cccctttgtaggacagagca & 9 & NM_002160.3 \\
\hline RPL13A & caagcggatgaacaccaac & tgtggggcagcatacctc & 28 & NM_012423.3 \\
\hline
\end{tabular}

\subsection{Statistical Analysis}

All values were reported as mean \pm standard deviation. Significant differences were judged using one-way analysis of variance (ANOVA) and the Student's t-test for pairwise comparison, considering $p$-values $<0.05$ statistically significant.

\section{Results}

\subsection{BM-MSC Aggregates Formation and Areas}

RGD-Cys-D1-PLLA nanopatterned substrates supported the adhesion of OA- and H-derived BM-MSCs. Cell aggregation was observed in all functionalized surfaces, revealing different aggregation patterns and sizes (Figure 2) and showed that an increase in RGD-Cys-D1 dendrimer concentration, up to a concentration of $2.5 \times 10^{-8}$, led to an increase in the cell aggregate area, decreasing for the highest concentration $\left(10^{-2}\right)$ (Figure 3$)$. Even though this trend failed to reach statistical significance $(p=0.07)$, these results are concordant with previous findings.
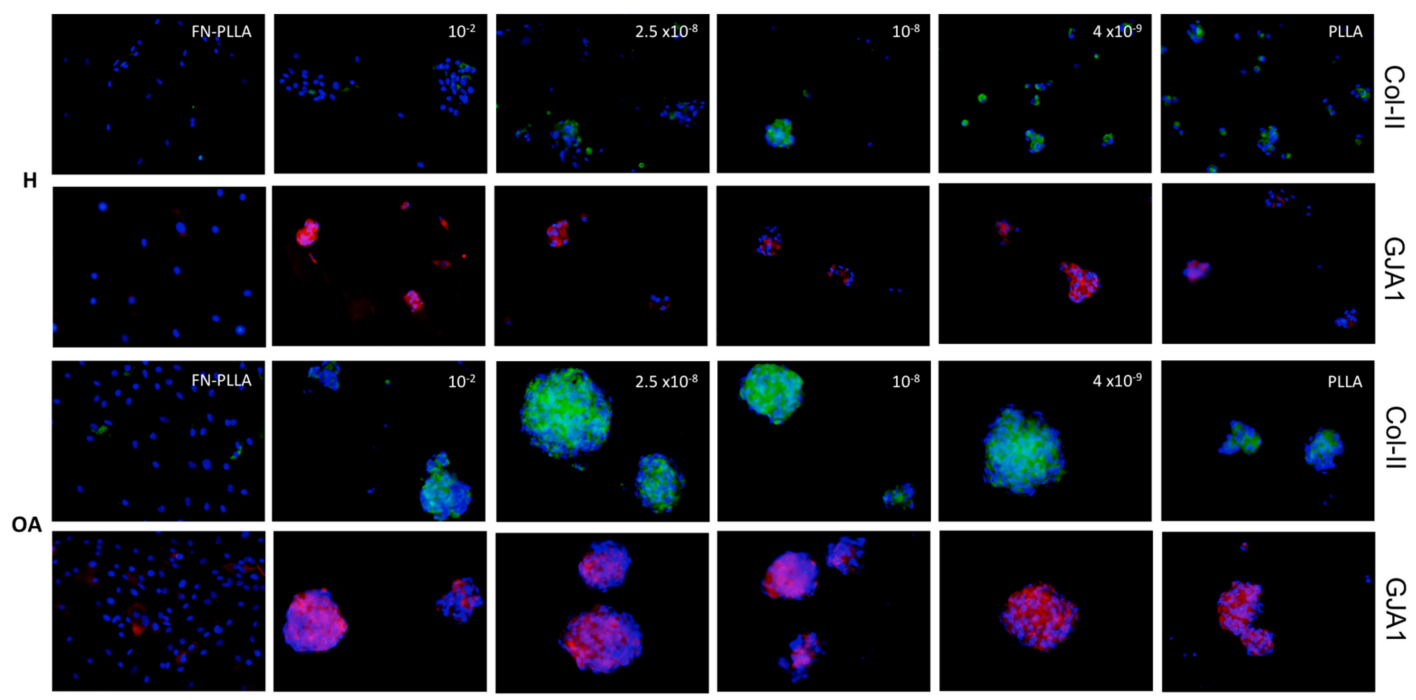

Figure 2. Collagen type-II (Col-II) and connexin 43 (expressed by the gap junction protein alpha 1, GJA1) immunofluorescence counterstained with DAPI, in healthy $(\mathrm{H})$ - and osteoarthritic (OA)-derived bone marrow mesenchymal stromal cells (BM-MSCs) aggregates formed after three days, under chondrogenic medium (CM), in RGD-Cys-D1 PLLA nanopatterned substrates $\left(10^{-2}, 2.5 \times 10^{-8}, 10^{-8}\right.$ and $4 \times 10^{-9}$ ), fibronectin-coated PLLA (Fn-PLLA), and untreated PLLA (PLLA). Scale bar: $200 \mu \mathrm{m}$.

Indeed, these results correlate with the increasing RGD density (surface adhesiveness) coated on PLLA substrates with dendrimers up to $2.5 \times 10^{-8}$, which decreases at $10^{-2}$, because dendrimers at this concentration have been shown to aggregate in solution and adsorb on the carriers as clusters 
rather than individual particles [14]. Moreover, this distinct behavior is consistent with a RGD nanospacing threshold value around $70 \mathrm{~nm}$, above which the cell adhesion process is delayed [26]. More specifically, H-derived MSC aggregate formation was observed in all conditions, except for Fn-PLLA (positive control), where cells were homogenously distributed in a monolayer.

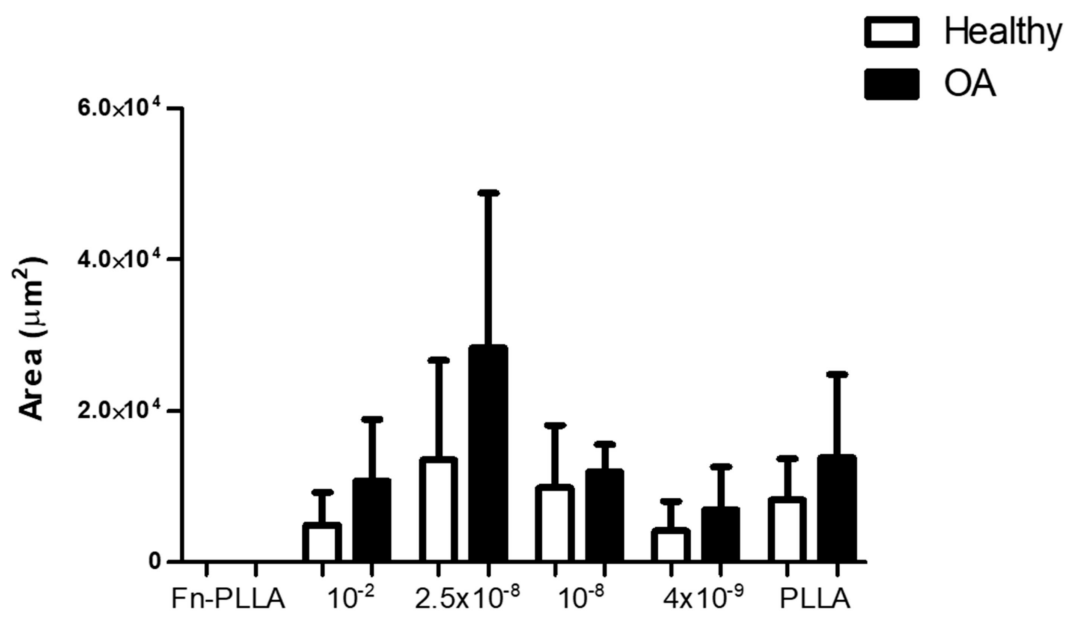

Figure 3. Area (units) of healthy- $(\mathrm{H})$ and osteoarthritic (OA)-derived BM-MSC aggregates formed in RGD-Cys-D1 PLLA nanopatterned substrates $\left(10^{-2}, 2.5 \times 10^{-8}, 10^{-8}\right.$, and $\left.4 \times 10^{-9}\right)$, fibronectin-coated PLLA (Fn-PLLA), or untreated PLLA (PLLA) after three days, under chondrogenic medium (CM). Values are given as the mean of at least three aggregates with standard deviation.

Nonetheless, cell aggregates were either small $\left(2.86 \times 10^{4}\right.$ to $\left.0.39 \times 10^{4} \mu \mathrm{m}^{2}\right)$ or appeared to be in the process of cell aggregation, but not fully compact (as can be distinctly observed for the $10^{-2}$ and $2.5 \times 10^{-8}$ concentrations, top images, Figure 2). These values were in the range of the ones previously found for AT-MSCs [14]. On the other hand, OA-derived MSCs formed bigger $\left(4.14 \times 10^{4}\right.$ to $0.22 \times 10^{4} \mu \mathrm{m}^{2}$ ) and compact aggregates.

Untreated PLLA substrates (negative control) induced cell aggregation, more marked for OA-derived MSCs. In the negative control of chondrogenesis (basal medium) in Fn-PLLA substrates, cells were disposed in a confluent monolayer and did not form aggregates (Figure S1). Moreover, differences observed between the aggregate areas from OA- versus H-derived BM-MSCs seem to be independent of the substrate used, as this difference could also be appreciated in BM-MSCs pellets in the 3D conventional culture system (Figure 4).

a)

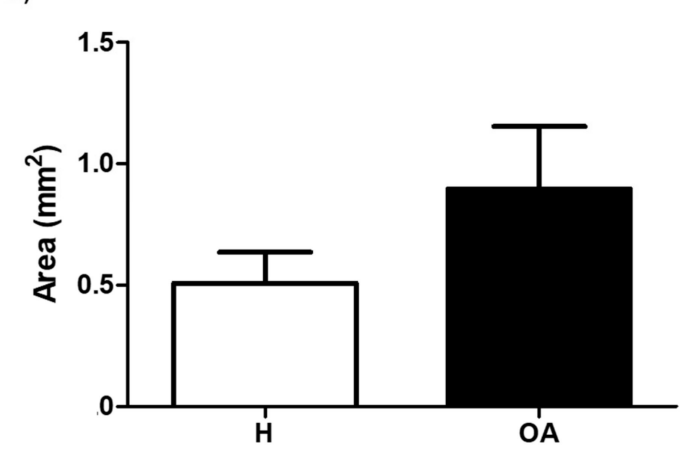

b)

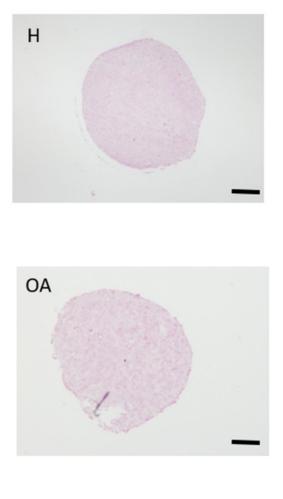

Figure 4. (a) Area $\left(\mathrm{mm}^{2}\right)$ of $\mathrm{H}$ - and OA-derived BM-MSCs pellets in 3D-pellet conventional system after three days, under CM. Values are given as the mean of at least three donors with standard deviation. (b) Hematoxylin-Eosin (H-E) staining. Scale bar: $200 \mu \mathrm{m}$. 


\subsection{Molecular Expression and Protein Synthesis}

Figure 5 represents the mRNA relative expression of cell aggregates from $\mathrm{H}$ - and OA-derived MSCs under the different conditions. Early chondrogenic markers, SOX9 and TNC, were upregulated for higher RGD-Cys-D1 dendrimer concentrations $\left(2.5 \times 10^{-8}\right.$ in $\mathrm{H}$ and $10^{-2}$ in OA, although only significant for $10^{-2}$ vs. $10^{-8}$ and $10^{-2}$ vs. $2.5 \times 10^{-8}$, in the latest) as well as in comparison with the controls (untreated PLLA and Fn-PLLA). COL1A1 expression followed a similar pattern such as other genes studied in H-derived cell aggregates, with an upregulation at $2.5 \times 10^{-8}$, whilst in OA-derived cell aggregates, COL1A1 expression was practically unaltered and downregulated for all conditions when compared to Fn-PLLA.

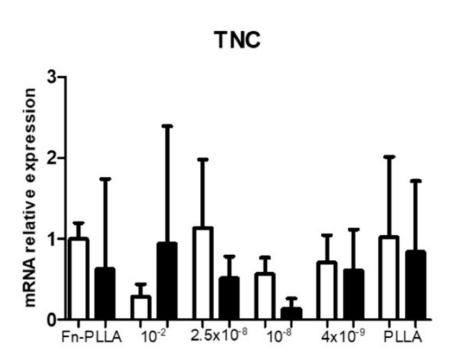

COL1A1

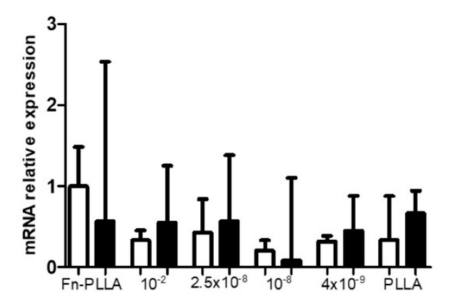

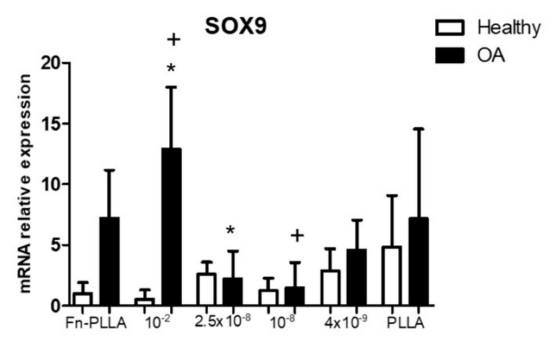

COL2A1

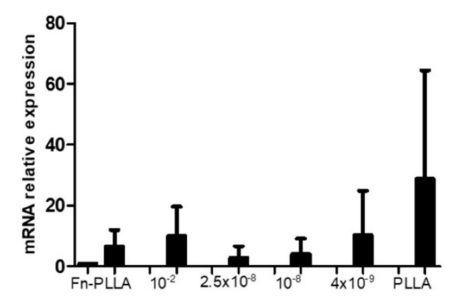

GJA1

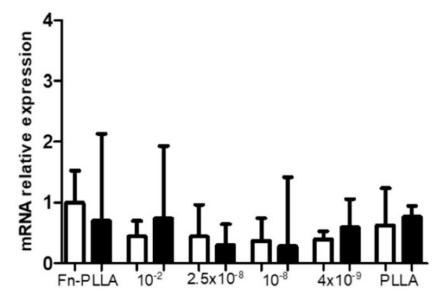

Figure 5. mRNA relative expression of TNC, SOX9, COL1A1, COL2A1, and GJA1 from H-(white) and OA-(black) derived BM-MSC aggregates formed in RGD-Cys-D1 PLLA nanopatterned substrates $\left(10^{-2}, 2.5 \times 10^{-8}, 10^{-8}\right.$, and $\left.4 \times 10^{-9}\right)$, fibronectin-coated PLLA (Fn-PLLA), and untreated PLLA (PLLA), after three days, under $\mathrm{CM}$. Values are given as the mean of three donors with standard deviation.

* + indicate $p<0.05$.

The COL2A1 chondrogenic marker was only found expressed in OA-derived cell aggregates, with a similar expression trend as found for TNC and SOX9. Col-II protein expression was strongly synthesized when compared to the poorly stained $\mathrm{H}$-derived cell aggregates (Figure 2). Even though the mRNA transcript level of the cartilage-related COL2A1 gene was non-detectable for the latest cells, the col-II protein was found accumulated in the extracellular matrix, which might be explained by the fact that other mechanisms regulate the protein's abundance. As mRNA is prone to degradation, it is possible that mRNA has a rapid turnover while its protein has a higher half-life and remains accumulated [27].

GJA1 expression was upregulated for $2.5 \times 10^{-8}$ regarding other dendrimer concentrations in $\mathrm{H}$-derived cell aggregates, whilst showing a downregulation at this concentration in OA-derived cells. Cx43 was also observed at the protein level in both OA- and H-derived cell aggregates. In addition, OA-derived MSCs seeded in Fn-PLLA were poorly positive regarding H-derived cells that were negative (Figure 2). The same behavior was observed in the basal medium (Figure S1).

In the pellet system, comparison between $\mathrm{H}$ - and OA-derived MSCs indicated a downregulation of TNC and COL1A1 whilst an upregulation of GJA1 was observed. SOX9 showed a similar trend as observed for cell-aggregates formed in the different nanopatterns. In addition, no COL2A1 expression was detected (Figure 6). 
TNC
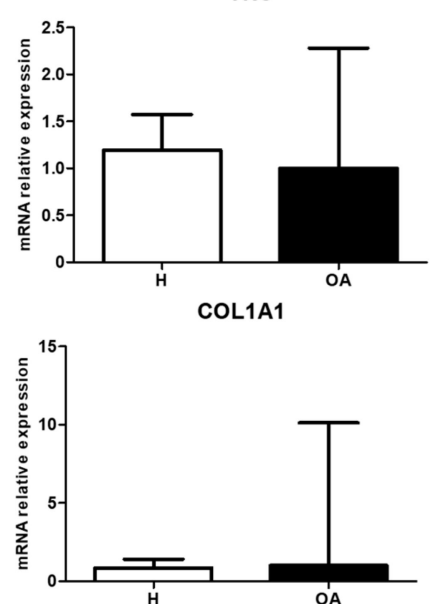

sox9
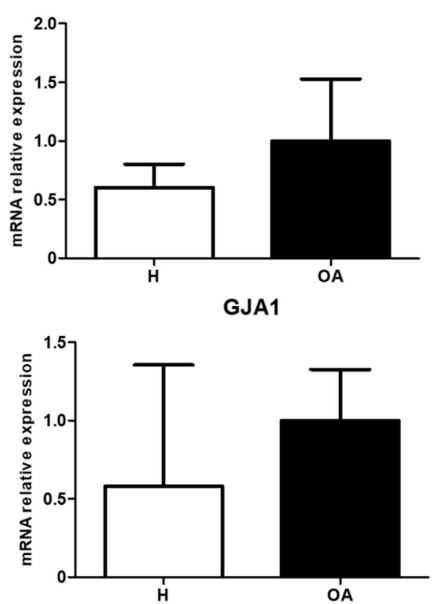

Figure 6. mRNA relative expression of TNC, SOX9, COL1A1, and GJA1 from H-(white) and OA-(black) derived BM-MSCs pellets, after three days, under CM. Values are given as the mean of three donors with standard deviation.

Regarding protein expression in the pellets 3D-system, H-derived MSCs showed weak col-II staining that was limited to single cells and the absence of $\mathrm{Cx} 43$, whilst both markers were absent in pellets from OA-derived MSCs (Figure 7a). Nonetheless, SO staining, indicative of proteoglycans synthesis was found increased in the latest (Figure 7b).

a)
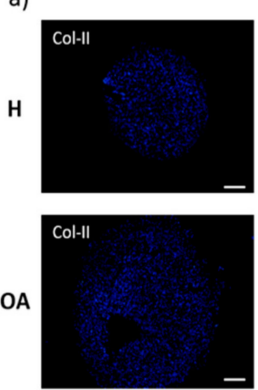
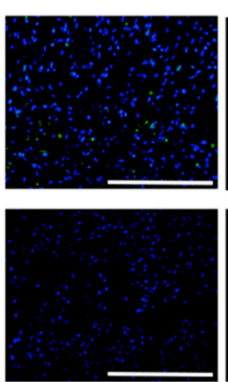
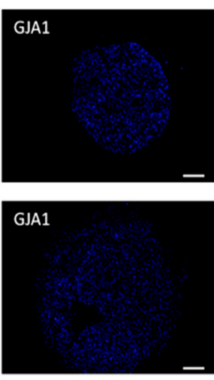

b)

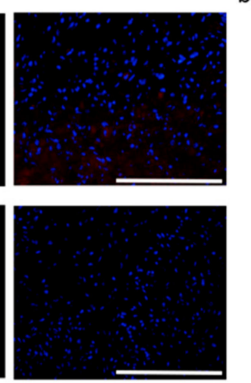

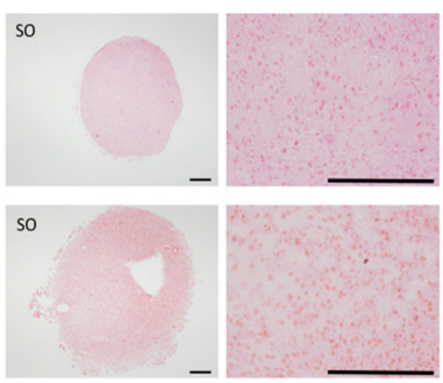

Figure 7. (a) Col-II and GJA1 immunofluorescence counterstained with DAPI. (b) SO staining for proteoglycans in H- and OA-derived BM-MSCs pellets, after three days, under CM. Scale bar: $200 \mu \mathrm{m}$.

\section{Discussion}

Cell condensation is a pivotal step during the early stages of chondrogenic differentiation. In this study, controlled nanopatterning of RGD-Cys-D1 over PLLA were used as bioactive substrates in order to evaluate RGD local surface density in the early chondrogenic differentiation of MSCs isolated from OA patients. Previous work demonstrated that tunable local RGD-Cys-D1 densities could be obtained as a function of the initial RGD-Cys-D1 dendrimer concentration, creating uneven distribution patterns of these first generation dendrimers, on PLLA coated glass surfaces [12]. Thereafter, together with our co-workers, we have shown that RGD-Cys-D1 PLLA nanopatterned substrates supported AT-MSC early chondrogenesis by stimulating the aggregation of the cells, followed by the maturation of focal adhesions that formed between them [14].

Whilst AT-MSCs are widely used and accepted, cellular models that can better resemble pathologic conditions are preferred, so we hereby intended to replicate the study conditions using human BM-MSC derived from $\mathrm{OA}$ and $\mathrm{H}$ donors. In addition, previous results from our group focused on cell-to-cell contact and cell communication in cartilage explant models showed higher expression of GJA1 in OA [20], this being associated with chondrocyte dedifferentiation [28]. Therefore, we intended to verify 
if there could be a dependency on connexin 43 and collagen-type II during a pre-chondrogenic phase of the differentiation process.

We found that already within three days after chondrogenic induction, MSCs seeded in RGD-Cys-D1 PLLA nanopatterned substrates condensed into cell aggregates and started a chondrogenic differentiation process (more pronounced for OA-derived MSCs). This was shown to be related to the presence and density of RGD-Cys-D1, suggesting $2.5 \times 10^{-8}$ as an optimal dendrimer concentration, with higher expression of the early chondrogenic marker SOX9, which is consistent with previous observations using other MSC sources [14]. Moreover, our results support a higher specificity of RGD peptide compared with Fn-PLLA, having the latest induced a monolayer-type behavior and no observed aggregation (Figures 2 and 3 ).

In addition, the cell-protein-material interface has been reported to affect cell migratory behavior, and differences in fibronectin conformation impact cell responses [29-31]. Even though we observed aggregation in untreated PLLA, this might be related to the polymer's hydrophobicity, which causes cells to cluster in the presence of a chondrogenic medium, as reported for other positively charged polymers [32,33]. Other in-house studies using PLLA coated surfaces under basal medium showed no cell aggregation (data not shown).

Moreover, RGD-Cys-D1 PLLA nanopatterned substrates favored chondrogenesis regarding the conventional pellet culture system, supported by the synthesis of collagen type-II and an increased cell-to-cell contact network given by the presence of connexin- 43 , both on the gene (Figures 5 and 7 ) and protein levels (Figures 2 and 6), which could support the role of RGD-mediated adhesion via integrins that trigger cell arrangement and size, favoring a pre-chondrogenic state on cell aggregates [34-38]. This also supports previous findings [12,14].

Another interesting outcome of this study were the differences found between $\mathrm{H}$ - and OA-derived MSCs. On one hand, the increased expression of both GJA1 and COL2A1 in OA-derived MSC was in disagreement with other studies that showed a link between overexpression of GJA1 in OA chondrocytes and a compromised chondrogenic capability [20]. On the other hand, the downregulation of COL1A1 and GJA1 and absence of COL2A1 in H-derived aggregates may indicate an early migration phase that is RGD-dependent in which cells resemble a more undifferentiated state [34,39,40].

Furthermore, differences found could be related to the expression of different patterns of membrane proteins present in either OA- or H-derived cells, although these have not been considered in this study [41-43]. Finally, both OA and aging effects have been shown to impact the chondrogenic potential of MSCs. Even though OA-derived cells have reportedly been demonstrated to undergo chondrogenesis [44], these have rarely shown outperformance, as opposed to our findings. Nonetheless, there is still controversy and a need for studies using clearly defined OA progression criteria and age-matched control subject groups that can further deepen our knowledge for their clinical relevance.

\section{Conclusions}

The results obtained showed that RGD-Cys-D1 PLLA nanopatterned substrates supported the formation of pre-chondrogenic condensates from OA- and H-derived MSCs. OA-derived MSCs cultured in nanopatterned substrates formed bigger and more compact aggregates with improved collagen-II expression (both at gene and protein level) regarding either $\mathrm{H}$-derived MSCs cultured in nanopatterned substrates or cell pellet conventional culture system, which support the use of the hereby proposed RGD-Cys-D1 PLLA nanopatterned substrates over the conventional pellet system in disease models.

Supplementary Materials: The following are available online at http://www.mdpi.com/1996-1944/13/10/2247/s1, Figure S1: Col-II and GJA1 immunofluorescence counterstained with DAPI, in H- and OA-derived BM-MSCs for three days under basal medium in fibronectin-coated PLLA (Fn-PLLA). Scale bar: $200 \mu \mathrm{m}$.

Author Contributions: C.R.-P., J.M., and F.J.B. conceptualized the research and C.R.-P. performed the experiments and data analyses; Y.V. and E.P.-I. designed and produced the RGD-functionalized dendrimers. I.C., A.L., and J.S. helped with the cell experiments and data analysis. J.A.A. and J.B. discussed the experiments based on dendrimer 
nanopatterning. C.R.-P. and J.M. wrote the manuscript with contributions from F.J.B., A.L., I.C., J.S., Y.V., E.P.-I., J.A.A., and J.B. All authors have read and agreed to the published version of the manuscript.

Funding: This research was funded by the CIBER-BBN Intramural Projects Program (CHONDRONANONET). CIBER-BBN is a national initiative of ISCIII. The authors further acknowledge funds from the INTERREG V cooperation program for Spain-Portugal (POCTEP) 2014-2020 project (0245_IBEROS_1_E), MINECO (CTQ2016-75870-P), Junta de Andalucía (UMA18-FEDERJA-007), CERCA Program, and by the Commission for Universities and Research of the Department of Innovation, Universities, and Enterprise of the Generalitat de Catalunya (2017 SGR 1079). This work was developed in the context of AdvanceCat with the support of ACCIÓ 17 (Catalonia Trade and Investment; Generalitat de Catalunya) under the Catalonian ERDF operational program 2014-2020. C.R.P. acknowledges support from the i-PFIS Doctoral Program (IFI15/00151) funded by ISCIII. I.C. acknowledges support from MINECO through the Subsidies for Predoctoral Contracts for the Training of Doctors open call, co-funded by the European Social Fund (2016).

Acknowledgments: We would like to acknowledge the Histology Platform and the Cell Culture Lab from GIR for their technical assistance.

Conflicts of Interest: The authors declare no conflicts of interest.

\section{References}

1. Kraus, V.B.; Blanco, F.J.; Englund, M.; Karsdal, M.A.; Lohmander, L.S. Call for standardized definitions of osteoarthritis and risk stratification for clinical trials and clinical use. Osteoarthr. Cartil. 2015, 23, $1233-1241$. [CrossRef]

2. Vinatier, C.; Guicheux, J. Cartilage tissue engineering: From biomaterials and stem cells to osteoarthritis treatments. Ann. Phys. Rehabil. Med. 2016, 59, 139-144. [CrossRef]

3. McMurray, R.J.; Dalby, M.J.; Tsimbouri, P.M. Using biomaterials to study stem cell mechanotransduction, growth and differentiation. J. Tissue Eng. Regen. Med. 2015, 9, 528-539. [CrossRef]

4. Dobbenga, S.; Fratila-Apachitei, L.E.; Zadpoor, A.A. Nanopattern-induced osteogenic differentiation of stem cells - A systematic review. Acta Biomater. 2016, 46, 3-14. [CrossRef] [PubMed]

5. Mashinchian, O.; Turner, L.A.; Dalby, M.J.; Laurent, S.; Shokrgozar, M.A.; Bonakdar, S.; Imani, M.; Mahmoudi, M. Regulation of stem cell fate by nanomaterial substrates. Nanomedicine 2015, 10, 829-847. [CrossRef] [PubMed]

6. Wang, X.; Yan, C.; Ye, K.; He, Y.; Li, Z.; Ding, J. Effect of RGD nanospacing on differentiation of stem cells. Biomaterials 2013, 34, 2865-2874. [CrossRef]

7. Kang, J.; Park, H.M.; Kim, Y.W.; Kim, Y.; Varghese, S.; Seok, H.K.; Kim, Y.G.; Kim, Y.H. Control of mesenchymal stem cell phenotype and differentiation depending on cell adhesion mechanism. Eur. Cells Mater. 2016, 28, 387-403. [CrossRef]

8. Medda, R.; Helth, A.; Herre, P.; Pohl, D.; Rellinghaus, B.; Perschmann, N.; Neubauer, S.; Kessler, H.; Oswald, S.; Eckert, J. Investigation of early cell-surface interactions of human mesenchymal stem cells on nanopatterned-type titanium-niobium alloy surfaces. Interface Focus 2013, 4, 20130046-20130046. [CrossRef]

9. Glennon-Alty, L.; Williams, R.; Dixon, S.; Murray, P. Induction of mesenchymal stem cell chondrogenesis by polyacrylate substrates. Acta Biomater. 2013, 9, 6041-6051. [CrossRef]

10. Nemeth, C.L.; Janebodin, K.; Yuan, A.E.; Dennis, J.E.; Reyes, M.; Kim, D.H. Enhanced Chondrogenic Differentiation of Dental Pulp Stem Cells Using Nanopatterned PEG-GelMA-HA Hydrogels. Tissue Eng. Part A 2014, 20, 2817-2829. [CrossRef] [PubMed]

11. Pericet-Camara, R.; Cahill, B.P.; Papastavrou, G.; Borkovec, M. Nano-patterning of solid substrates by adsorbed dendrimers. Chem. Commun. 2007, 266-268. [CrossRef]

12. Lagunas, A.; Castaño, A.G.; Artés, J.M.; Vida, Y.; Collado, D.; Pérez-Inestrosa, E.; Gorostiza, P.; Claros, S.; Andrades, J.A.; Samitier, J. Large-scale dendrimer-based uneven nanopatterns for the study of local arginine-glycine-aspartic acid (RGD) density effects on cell adhesion. Nano Res. 2014, 7, 399-409. [CrossRef]

13. Xiong, J.P. Crystal Structure of the Extracellular Segment of Integrin alpha Vbeta 3 in Complex with an Arg-Gly-Asp Ligand. Science 2002, 296, 151-155. [CrossRef] [PubMed]

14. Lagunas, A.; Tsintzou, I.; Vida, Y.; Collado, D.; Pérez-Inestrosa, E.; Rodríguez Pereira, C.; Magalhaes, J.; Andrades, J.A.; Samitier, J. Tailoring RGD local surface density at the nanoscale toward adult stem cell chondrogenic commitment. Nano Res. 2017, 10, 1959-1971. [CrossRef]

15. Singh, P.; Schwarzbauer, J.E. Fibronectin and stem cell differentiation-Lessons from chondrogenesis. J. Cell Sci. 2012, 125, 3703-3712. [CrossRef] [PubMed] 
16. Kwon, H.J.; Ohmiya, Y.; Honma, K.I.; Honma, S.; Nagai, T.; Saito, K.; Yasuda, K. Synchronized ATP oscillations have a critical role in prechondrogenic condensation during chondrogenesis. Cell Death Dis. 2012, 3, e278. [CrossRef]

17. Plotkin, L.I.; Stains, J.P. Connexins and pannexins in the skeleton: Gap junctions, hemichannels and more. Cell. Mol. Life Sci. 2015, 72, 2853-2867. [CrossRef]

18. Zhang, Y.D.; Zhao, S.C.; Zhu, Z.S.; Wang, Y.F.; Liu, J.X.; Zhang, Z.C.; Xue, F. Cx43-and Smad-Mediated TGF- $\beta$ /BMP Signaling Pathway Promotes Cartilage Differentiation of Bone Marrow Mesenchymal Stem Cells and Inhibits Osteoblast Differentiation. Cell. Physiol. Biochem. 2017, 42, 1277-1293. [CrossRef]

19. Loty, S.; Foll, C.; Forest, N.; Sautier, J.M. Association of enhanced expression of gap junctions with in vitro chondrogenic differentiation of rat nasal septal cartilage-released cells following their dedifferentiation and redifferentiation. Arch. Oral Biol. 2000, 45, 843-856. [CrossRef]

20. Mayan, M.D.; Carpintero-Fernandez, P.; Gago-Fuentes, R.; Martinez-De-Ilarduya, O.; Wang, H.Z.; Valiunas, V.; Brink, P.; Blanco, F.J. Human articular chondrocytes express multiple gap junction proteins: Differential expression of connexins in normal and osteoarthritic cartilage. Am. J. Pathol. 2013, 182, 1337-1346. [CrossRef]

21. Schrobback, K.; Klein, T.J.; Woodfield, T.B.F. The Importance of Connexin Hemichannels During Chondroprogenitor Cell Differentiation in Hydrogel Versus Microtissue Culture Models. Tissue Eng. Part A 2015, 21, 1785-1794. [CrossRef] [PubMed]

22. Brandt, K.D.; Fife, R.S.; Braunstein, E.M.; Katz, B. Radiographic grading of the severity of knee osteoarthritis: Relation of the Kellgren and Lawrence grade to a grade based on joint space narrowing, and correlation with arthroscopic evidence of articular cartilage degeneration. Arthritis Rheum. 1991, 34, 1381-1386. [CrossRef] [PubMed]

23. Hermida-Gómez, T.; Fuentes-Boquete, I.; Gimeno-Longas, M.J.; Muiños-López, E.; Díaz-Prado, S.; de Toro, F.J.; Blanco, F.J. Bone Marrow Cells Immunomagnetically Selected For CD271+ Antigen Promote In Vitro the Repair of Articular Cartilage Defects. Tissue Engineering Part A 2011, 17, 1169-1179. [CrossRef] [PubMed]

24. Solchaga, L.A.; Penick, K.J.; Welter, J.F. Chondrogenic Differentiation of Bone Marrow-Derived Mesenchymal Stem Cells: Tips and Tricks. Methods Mol. Biol. 2011, 698, 253-278. [CrossRef]

25. Schindelin, J.; Arganda-Carreras, I.; Frise, E.; Kaynig, V.; Longair, M.; Pietzsch, T.; Preibisch, S.; Rueden, C.; Saalfeld, S.; Schmid, B.; et al. Fiji: An open source platform for biological image analysis. Nat. Methods 2012, 9, 676-682. [CrossRef]

26. Casanellas, I.; Lagunas, A.; Tsintzou, I.; Vida, Y.; Collado, D.; Pérez-Inestrosa, E.; Rodríguez-Pereira, C.; Magalhaes, J.; Gorostiza, P.; Andrades, J.A.; et al. Dendrimer-based Uneven Nanopatterns to Locally Control Surface Adhesiveness: A Method to Direct Chondrogenic Differentiation. J. Vis. Exp. 2018, 131. [CrossRef]

27. Sonenberg, N.; Hinnebusch, A.G. Regulation of translation initiation in eukaryotes: Mechanisms and biological targets. Cell 2009, 136, 731-745. [CrossRef]

28. Krishnan, H.; Miller, W.T.; Blanco, F.J.; Goldberg, G.S. Src and podoplanin forge a path to destruction. Drug Discov. Today 2019, 24, 241-249. [CrossRef]

29. Dolatshahi-Pirouz, A.; Jensen, T.; Kraft, D.C.; Foss, M.; Kingshott, P.; Hansen, J.L.; Larsen, A.N.; Chevallier, J.; Besenbacher, F. Fibronectin adsorption, cell adhesion, and proliferation on nanostructured tantalum surfaces. ACS Nano 2010, 4, 2874-2882. [CrossRef]

30. Lin, M.; Mao, S.; Wang, J.; Xing, J.; Wang, Y.; Cai, K.; Luo, Y. Adsorption force of fibronectin controls transmission of cell traction force and subsequent stem cell fate. Biomaterials 2018, 162, 170-182. [CrossRef]

31. Grigoriou, E.; Cantini, M.; Dalby, M.J.; Petersen, A.; Salmeron-Sanchez, M. Cell migration on material-driven fibronectin microenvironments. Biomater. Sci. 2017, 5, 1326-1333. [CrossRef]

32. Brammer, K.S.; Choi, C.; Frandsen, C.J.; Oh, S.; Jin, S. Hydrophobic nanopillars initiate mesenchymal stem cell aggregation and osteo-differentiation. Acta Biomater. 2011, 7, 683-690. [CrossRef] [PubMed]

33. Sart, S.; Tsai, A.C.; Li, Y.; Ma, T. Three-Dimensional Aggregates of Mesenchymal Stem Cells: Cellular Mechanisms, Biological Properties, and Applications. Tissue Eng. Part B: Rev. 2013, 20, 365-380. [CrossRef] [PubMed]

34. Cheng, T.; Maddox, N.C.; Wong, A.W.; Rahnama, R.; Kuo, A.C. Comparison of gene expression patterns in articular cartilage and dedifferentiated articular chondrocytes. J. Orthop. Res. 2012, 30, 234-245. [CrossRef] [PubMed]

35. Mackie, E.J.; Murphy, L.I. The role of tenascin-C and related glycoproteins in early chondrogenesis. Microsc. Res. Tech. 1998, 43, 102-110. [CrossRef] 
36. Murphy, L.I.; Fischer, D.; Chiquet-Ehrismann, R.; Mackie, E.J. Tenascin-C induced stimulation of chondrogenesis is dependent on the presence of the C-terminal fibrinogen-like globular domain. FEBS Lett. 2000, 480, 189-192. [CrossRef]

37. Bhumiratana, S.; Eton, R.E.; Oungoulian, S.R.; Wan, L.Q.; Ateshian, G.A.; Vunjak-Novakovic, G. Large, stratified, and mechanically functional human cartilage grown in vitro by mesenchymal condensation. Proc. Natl. Acad. Sci. USA 2014, 111, 6940-6945. [CrossRef]

38. DeLise, A.M.; Fischer, L.; Tuan, R.S. Cellular interactions and signaling in cartilage development. Osteoarthr. Cartil. 2000, 8, 309-334. [CrossRef]

39. Lefebvre, V.; Smits, P. Transcriptional control of chondrocyte fate and differentiation. Birth Defects Res. Part C - Embryo Today: Rev. 2005, 75, 200-212. [CrossRef]

40. Xu, J.; Wang, W.; Ludeman, M.; Cheng, K.; Hayami, T.; Lotz, J.C.; Kapila, S. Chondrogenic Differentiation of Human Mesenchymal Stem Cells in Three-Dimensional Alginate Gels. Tissue Eng. Part A 2008, 14, 667-680. [CrossRef]

41. Wu, J.; Liu, W.; Bemis, A.; Wang, E.; Qiu, Y.; Morris, E.A.; Flannery, C.R.; Yang, Z. Comparative proteomic characterization of articular cartilage tissue from normal donors and patients with osteoarthritis. Arthritis Rheum. 2007, 56, 3675-3684. [CrossRef] [PubMed]

42. Lourido, L.; Calamia, V.; Mateos, J.; Fernández-Puente, P.; Fernández-Tajes, J.; Blanco, F.J.; Ruiz-Romero, C. Quantitative proteomic profiling of human articular cartilage degradation in osteoarthritis. J. Proteome Res. 2014, 13, 6096-6106. [CrossRef] [PubMed]

43. Li, M.; Zhi, L.; Zhang, Z.; Bian, W.; Qiu, Y. Identification of potential target genes associated with the pathogenesis of osteoarthritis using microarray based analysis. Mol. Med. Rep. 2017, 16, 2799-2806. [CrossRef] [PubMed]

44. Brady, K.; Dickinson, S.C.; Hollander, A.P. Changes in Chondrogenic Progenitor Populations Associated with Aging and Osteoarthritis. Cartilage 2015, 6 (Suppl. 2), 30S-35S. [CrossRef]

(C) 2020 by the authors. Licensee MDPI, Basel, Switzerland. This article is an open access article distributed under the terms and conditions of the Creative Commons Attribution (CC BY) license (http://creativecommons.org/licenses/by/4.0/). 